\title{
Multiflash whistlers in ELF-band observed at low latitude
}

\author{
Ashutosh K. Singh ${ }^{1}$, K. K. Singh ${ }^{1}$, S. B. Singh ${ }^{1,2}$, A. K. Singh ${ }^{1}$, and Lalmani ${ }^{3}$ \\ ${ }^{1}$ Department of Physics, Banaras Hindu University, Varanasi, India \\ ${ }^{2}$ Department of Physics, Maharaja College, V. K. S. University, Ara, Bihar, India \\ ${ }^{3}$ Department of Physics, National Institute of Technology, Srinagar, Kashmir, India
}

Received: 13 May 2010 - Revised: 15 September 2010 - Accepted: 11 October 2010 - Published: 10 January 2011

\begin{abstract}
Multiflash whistler-like event in the ELF-band, observed during March 1998 at low latitude station Jammu, is reported. The most prominent feature of these events is the multiflash nature along with the decrease in frequency within a very short span of time resembling similar to terrestrial whistlers. The events have a significantly smaller time duration $(0.5-3.5 \mathrm{~s})$ than those reported earlier from high, mid and low latitudes and also display a diurnal maximum occurring around 09:30 $\mathrm{h}$ (IST). There have been similar reportings from other latitudes, but whistlers in the ELF-band with a multiflash nature along with a precursor emission have never been reported. Lightning seems to be the dominant source for the ELF whistlers reported here.
\end{abstract}

Keywords. Magnetospheric physics (Energetic particles, trapped)

\section{Introduction}

Studies on lightning generated whistlers show that the terrestrial whistlers have a frequency in the range $3-10 \mathrm{kHz}$ with a peak occurrence of around $5 \mathrm{kHz}$ (Helliwell, 1965). Extremely low frequency (ELF: 3-3000 Hz) waves have been associated with a range of magnetosphere-ionosphere coupling phenomena as well as meteorological phenomena (Magunia, 1996; Cummer et al., 1998). Heacock (1974), Sentman and Ehring (1994), Wang et al. (2005) and Kim et al. (2006) have reported ELF emissions/whistlers at much lower frequencies between 40-200 Hz. Heacock (1974) discovered dispersive emission in the ELF-band near Fairbanks, Alaska (geomag. latitude $65^{\circ} \mathrm{N}$, long. $256^{\circ} \mathrm{E}$ ) at high latitude. These emissions were detected to possess a frequency range similar to magnetosheath lion roar (Smith et al., 1967)

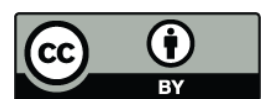

Correspondence to: A. K. Singh

(abhay_s@ rediffmail.com) but their spectral forms are quite different (Heacock, 1974). Sentman and Ehring's (1994) measurements at California reported whistler-like events with frequencies laying in ELFband, along with the dispersion characteristics and the local time occurrence distribution similar to the events detected by Heacock (1974). Recently, Wang et al. (2005) and Kim et al. (2006) also reported a similar type of ELF phenomena.

In this paper, we report first low latitude observation of multiflash and multipath ELF whistlers event. Two and more whistlers closely associated in time, but having different sources are known as multiflash whistlers while a whistler with two or more components, each of which have traversed a different path through the ionosphere is known as multipath whistler (Helliwell, 1965). These events are similar to those observed previously at mid and low latitude by Sentman and Ehring (1994) and Wang et al. (2005) in some aspects but no observation report multiflash nature of ELF whistlers. Also they are very short in duration and have a dispersion resembling terrestrial (VLF) whistlers contrary to the previously reported ELF whistlers. Moreover, ELF whistlers in our observations are detected in a higher ELF-band or up to $3 \mathrm{kHz}$ in range along with a group of whistlers with an upper cut-off frequency of only $900 \mathrm{~Hz}$.

Section 2 briefly describes the experimental apparatus used to record the data and the data processing technique used to detect ELF whistlers. The observational results of ELF whistlers are described in Sect. 3. The discussions of the results, with a possible source generation mechanism, are described in Sect. 4 and finally Sect. 5 summarizes the conclusions of the study.

\section{Instrumentation and data analysis}

Observations reported in this paper were detected at a low noise site in the northern part of India, Jammu (geomag. lat., $19^{\circ} 26^{\prime} \mathrm{N} ; L=1.17$ ) during the period from January-June

Published by Copernicus Publications on behalf of the European Geosciences Union. 


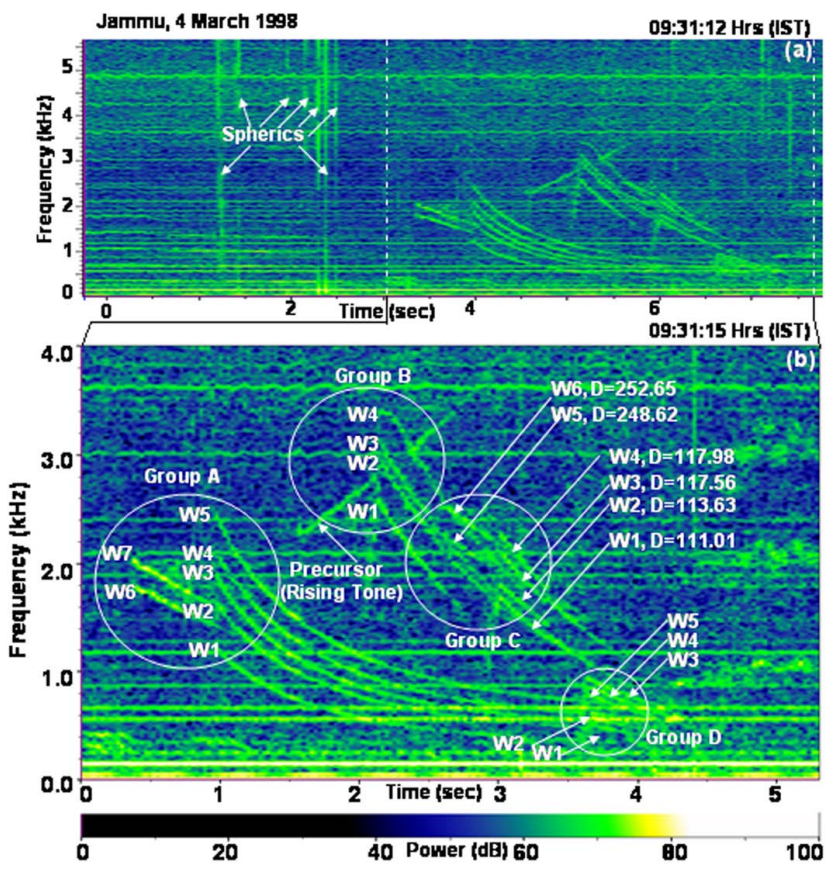

Fig. 1. (a) The frequency-time spectrogram of multiflash ELF whistlers observed in local daytime on 4 March 1998 at 09:31:12 IST at low latitude station Jammu. The corresponding causative spherics are marked by arrows. (b) The frequency-time spectrograms of enlarged four sets of above group of ELF whistlers starting at 09:31:15 IST along with a precursor emission which is marked by arrow.

1998. This site is away from industrial and other electromagnetic noises. The signals were detected on the standard whistlers observation equipment having a band width of $50 \mathrm{~Hz}-15 \mathrm{kHz}$ (Singh et al., 2009) consisting of T-type antenna which is $25 \mathrm{~m}$ in vertical length and $6 \mathrm{~m}$ long horizontally and $3.2 \mathrm{~mm}$ in diameter. The impedance is about $1 \mathrm{M} \Omega$. The antenna is rendered aperiodic with the help of a suitable $\mathrm{RC}$ network, to avoid any possible ringing effect. This is erected at a suitable distance from the main building to reduce the power line hum and any other type of man-made noises. The gain of the pre/main amplifier is varied from 0 to $40 \mathrm{~dB}$ to avoid overloading of the amplifier at the time of great ELF/VLF activity.

The observations were taken continuously both during day and night times. The ELF/VLF data were stored on the magnetic tapes, which were analysed on a digital sonograph available at the Department of Physics, Banaras Hindu University, Varanasi. The results of analysis show a number of whistlers and ELF/VLF emissions during the period of observation. Among the events observed only a few lie in the ELF-band and only one group of whistlers shown in Fig. 1 show the multiflash nature with a precursor emission in the ELF/VLF range with temporal fine structure having a very low cut-off frequency. The precursor emission is the rising tone emission that on rare occasion is observed to precede a whistler (Helliwell, 1965).

Whistler dispersion analysis using Eckersley-Story approximation (Helliwell, 1965) was used to determine the $L$ shell of propagation and the equatorial cold plasma density. In this method, the actual variation of frequency $(f)$ with time is plotted, which is called the dynamic spectra of the whistler. Again by plotting $1 / \sqrt{ } f$ versus time $(t)$, we obtain a straight line passing through the origin, the reciprocal of the slope of this line is called the dispersion, $D$ and is equal to the time of propagation multiplied by the square root of the frequency, $D=t \sqrt{ } f \mathrm{~s}^{1 / 2}$ (Helliwell, 1965).

Nose whistlers show rising and falling spectral tones simultaneously starting at a frequency called the nose frequency, $f_{\mathrm{n}}$. At frequencies above the "nose", the frequency of the whistler increases with time while below the nose the frequency decreases with time. To determine the nose frequency, we use the nose extension method of Dowden and Allcock (1971). In this method, the function $Q=1 / D$ is approximated by a linear function of frequency. Here we plot $Q$ as a function of frequency, the $Q(f)$ points fit closely to a straight line which intercepts the $Q=0$ axis at some value $f_{o}$ (zero- $Q$ frequency). Dowden and Allcock (1971) found that the nose whistler gave a mean ratio of zero- $Q$ frequency $\left(f_{o}\right)$ to observed nose frequency of $3.09 \pm 0.04$. This property was used to determine the nose frequency $\left(f_{\mathrm{n}}\right)$ of the whistlers, which do not exhibit nose by extrapolating the $Q(f)$ regression line obtained from $t(f)$ measurements within the available frequency range. Thus, $f_{\mathrm{n}}$ is determined by using the relation $f_{\mathrm{n}}=f_{o} / 3.1$ (Dowden and Allcock, 1971) and hence $f_{\mathrm{Heq}}$, the equatorial gyrofrequency along the field line, through the relation $f_{\mathrm{n}}=0.4 f_{\mathrm{Heq}}$ (Sazhin et al., 1992). The measured values were then used together with a diffusive equlibrium model of the cold plasma distribution along the field line (Angerami and Thomas, 1964) to infer the equatorial electron density $N_{\text {eq }}$. This analysis revealed the values of $L$ lying between 3.25 to 6.52 and $N_{\text {eq }}=96-323 \mathrm{~cm}^{-3}$, consistent with the empirical model of Carpenter and Anderson (1992).

\section{Observations}

The frequency-time spectrograms of ELF whistlers are shown in Fig. 1a. The corresponding causative spherics are marked by arrows. The four group sets of ELF whistlers are magnified and clearly shown in Fig. 1b. These were observed in winter local daytime on 4 March 1998 at 09:31:15 IST (Indian Standard Time) during a geomagnetically disturbed period. The magnetic activity during the period of observation was disturbed $\left(\sum \mathrm{Kp}=17_{+}\right)$. This peculiar event with a multiflash nature occurred only once during the whole period of observation. Time duration for various ELF whistlers and this unique multiflash ELF whistler event ranges from $0.5 \mathrm{~s}$ to $3.5 \mathrm{~s}$ much smaller than those in previous studies (Heacock, 
1974; Kim et al., 2006; Sentman and Ehring, 1994; Wang et al., 2005) which were around $40 \mathrm{~s}$ to $120 \mathrm{~s}$.

Group A of Fig. 1 shows the spectrograms of the first group set of ELF whistlers containing seven traces of onehop multipath high dispersion whistlers in the frequency range $\sim 400 \mathrm{~Hz}-2.4 \mathrm{kHz}$, which has travelled along different field lines (paths) having different dispersions (Singh et al., $2004 b$ ). From the dispersion analysis of these whistlers, it is found that the dispersion of whistlers marked W1 to W7 are in increasing order and are 101.28, 121.09, 121.22, 139.41, $152.79,200.47$ and $212.45 \mathrm{~s}^{1 / 2}$, respectively. Out of which whistlers W6 and W7 occur earlier to whistlers W1-W5 and form another pair of whistlers and are seen to have occurred to the left in frequency-time plane before whistlers W1-W5 and seems to touch whistler W2.

Group B of Fig. 1 illustrates one long intense precursor, crossing almost all the four whistler components of the second group set of ELF whistlers in the frequency ranges $\sim 1.6$ to $3.2 \mathrm{kHz}$. The corresponding dispersions of whistler components W1 to W4 of this second set are 108.69, 136.98, 136.90 and $140.84 \mathrm{~s}^{1 / 2}$, respectively.

Group C of Fig. 1 shows a group of ELF whistlers having temporal fine structure similar to those seen in Group A in the frequency range $\sim 800 \mathrm{~Hz}$ to $2.6 \mathrm{kHz}$. The first four whistlers of group C, W1 to W4 appear like two whistlers pairs: W1-W2 and W3-W4. Further out of these whistlers components W5 and W6 occur before W1-W4 and posses much higher dispersion than the other four whistlers. The dispersion of whistlers W1 to W6 is $111.01,113.63,117.56$, $117.98,248.62$, and $252.65 \mathrm{~s}^{1 / 2}$, respectively.

Finally, group D of Fig. 1 shows the fourth group set of ELF whistlers containing five whistler components in the frequency range of about $400 \mathrm{~Hz}-900 \mathrm{~Hz}$. From the dispersion analysis it is evident that dispersion of whistlers, W1 to W5 are in increasing order, 67.43, 72.63, 73.79, 81.84 and $86.38 \mathrm{~s}^{1 / 2}$, respectively.

Here we observe that there is a slight increase in the dispersion for the corresponding members of a multiple whistlers in each group as tabulated in Table 1. The dispersions of Group $\mathrm{C}$ whistlers have also been marked in Fig. 1 for a clearer view. The slight increase in the dispersion of successive multiple whistlers are due to an increase in ionization along the whistlers path produced by the discharge themselves (Hoffman, 1960; Clarence and O'Brien, 1961).

The occurrence rate of ELF whistlers with respect to local time is plotted in Fig. 2 and compared with the previous low latitude observation reported by Wang et al. (2005). All events occurred between $06: 00 \mathrm{~h}$ to $23: 00 \mathrm{~h}$ (IST) consistent with the previous observation. The maximum number of events observed around 09:00 to 10:00 h (IST) which is consistent with the low latitude observation by Wang et al. (2005). There appears to be a night to dawn gap, the same as that reported by Heacock (1974), Sentman and Ehring (1994) or Wang et al. (2005). There also appears an increased rate of occurrence from 20:00-23:00 h (IST), but no

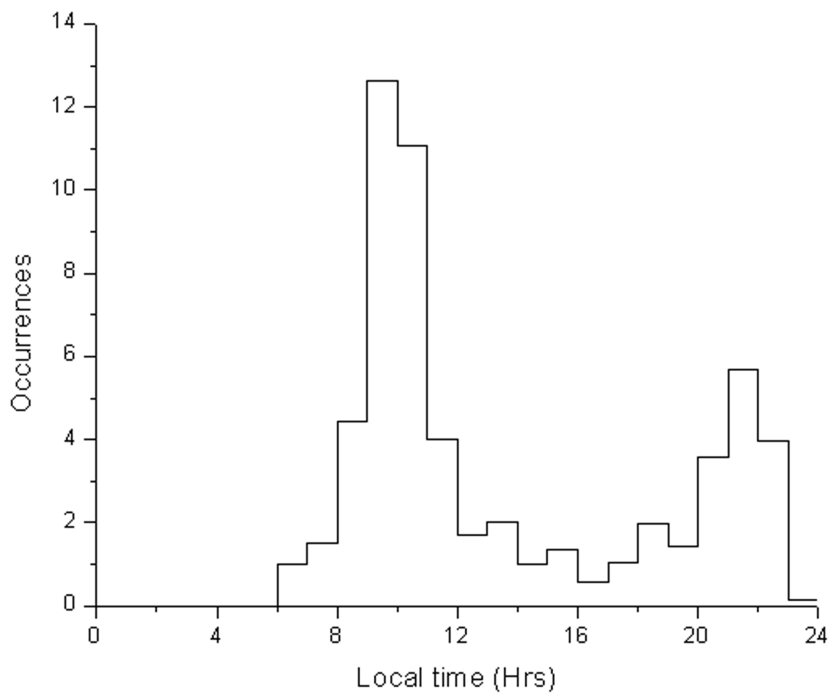

Fig. 2. The occurrence rate of ELF whistlers with respect to local time.

other peak is observed as reported by Wang et al. (2005) from low latitude. There was only one observation with a multiflash nature in the ELF-band along with a precursor emission around 09:31:16.5 h (IST).

Heacock (1974) found an anti-correlation between Ap index and occurrence. No significant correlation with Kp index and Ap index are observed by Sentman and Ehring (1994), Wang et al. (2005) or Kim et al. (2006). Our observation was detected during a geomagnetically disturbed period with planetary $\mathrm{K}$-index, $\mathrm{Kp}=17_{+}$. However, due to the small number of events (on most days with no events or only one event) and only single event with multiflash nature, we are unable to find any correlation between $\mathrm{Kp}$ index and ELF whistlers occurrence rate.

\section{Discussion}

There have been some similarities between our observed ELF/VLF events and those recorded in previous studies. These are the (1) daytime occurrence of the event with maximum around 09:30 $\mathrm{h}$ (IST), (2) frequency range of the event lying in ELF-band (although our observation lies near upper ELF-band). They differ in some aspects also, these are the (1) duration of event (only few seconds in our case while 40 to $120 \mathrm{~s}$ in previous observations), (2) Dispersion of the observed whistlers (only 67.4-252.6 s ${ }^{1 / 2}$ in our case while $>5000 \mathrm{~s}^{1 / 2}$ in previous observations). A complete table for the main results from Heacock (1974), Sentman and Ehring (1994), Wang et al. (2005), Kim et al. (2006) and the present paper are shown in Table 2. The signal pattern on frequency-time spectrogram, shown in Fig. 1a, suggests that they are generated from lightning source contrary to the previous suggested mechanism. The corresponding 
Table 1. Parameters estimated from the analysis of multiple whistlers observed at Jammu on 4 March 1998, 09:31:15 h.

\begin{tabular}{|c|c|c|c|c|}
\hline & Whistlers W & Dispersion $\mathrm{D}_{o}\left(\mathrm{~s}^{1 / 2}\right)$ & Nose frequency $f_{\mathrm{n}}(\mathrm{kHz})$ & L-Value \\
\hline \multirow{7}{*}{ Group A } & 1 & $101.28 \pm 1.25$ & 2.28 & 5.36 \\
\hline & 2 & $121.09 \pm 1.80$ & 2.92 & 4.83 \\
\hline & 3 & $121.22 \pm 1.00$ & 3.25 & 4.76 \\
\hline & 4 & $139.41 \pm 1.50$ & 3.62 & 4.59 \\
\hline & 5 & $152.79 \pm 1.25$ & 3.94 & 4.46 \\
\hline & 6 & $200.47 \pm 1.80$ & 6.73 & 3.73 \\
\hline & 7 & $212.45 \pm 2.90$ & 7.60 & 3.58 \\
\hline \multirow{4}{*}{ Group B } & 1 & $108.70 \pm 2.00$ & 5.35 & 4.03 \\
\hline & 2 & $136.99 \pm 2.50$ & 6.57 & 3.76 \\
\hline & 3 & $136.93 \pm 2.00$ & 6.76 & 3.73 \\
\hline & 4 & $140.84 \pm 2.20$ & 6.99 & 3.68 \\
\hline \multirow{6}{*}{ Group C } & 1 & $111.01 \pm 1.40$ & 3.65 & 4.58 \\
\hline & 2 & $113.64 \pm 1.50$ & 4.57 & 4.24 \\
\hline & 3 & $117.56 \pm 2.60$ & 4.78 & 4.18 \\
\hline & 4 & $117.98 \pm 2.60$ & 5.41 & 4.01 \\
\hline & 5 & $248.62 \pm 4.80$ & 9.12 & 3.37 \\
\hline & 6 & $252.65 \pm 5.00$ & 10.16 & 3.25 \\
\hline \multirow{5}{*}{ Group D } & 1 & $67.43 \pm 4.90$ & 1.50 & 6.15 \\
\hline & 2 & $72.63 \pm 2.00$ & 1.26 & 6.52 \\
\hline & 3 & $73.79 \pm 1.90$ & 1.89 & 5.69 \\
\hline & 4 & $81.42 \pm 3.50$ & 2.13 & 5.48 \\
\hline & 5 & $86.38 \pm 4.00$ & 2.36 & 5.29 \\
\hline
\end{tabular}

Table 2. Comparisons of ELF whistlers observations at different locations.

\begin{tabular}{|c|c|c|c|c|c|}
\hline Data source & $\begin{array}{l}\text { Heacock } \\
(1974)\end{array}$ & $\begin{array}{l}\text { Senman and Ehring } \\
\text { (1994) }\end{array}$ & $\begin{array}{l}\text { Wang et al. } \\
(2005)\end{array}$ & $\begin{array}{l}\text { Kim et al. } \\
(2006)\end{array}$ & This work \\
\hline Geographic Location & Auroral latitude $65^{\circ} \mathrm{N}$ & Mid-latitude $34^{\circ} \mathrm{N}$ & Low latitude $23.5^{\circ} \mathrm{N}$ & South Pole $-90^{\circ} \mathrm{N}$ & Jammu India $22^{\circ} 26^{\prime} \mathrm{N}$ \\
\hline Frequency & $40-200 \mathrm{~Hz}$ & $60-180 \mathrm{~Hz}$ & $60-100 \mathrm{~Hz}$ & $60-180 \mathrm{~Hz}$ & $200 \mathrm{~Hz}-3.2 \mathrm{kHz}$ \\
\hline Duration & $40 \mathrm{~s}-1.5 \mathrm{~min}$ & $40 \mathrm{~s}-1.5 \mathrm{~min}$ & $40 s-5 \min$ & $40 \mathrm{~s}-2 \mathrm{~min}$ & $0.5 \mathrm{~s}-3.5 \mathrm{~s}$ \\
\hline Dispersion & No echoes & No echoes & No echoes & No echoes & \\
\hline Local Time & Daytime maximum & Daytime maximum & Daytime maximum & Daytime maximum & Daytime maximum \\
\hline Correlation with $\mathrm{Kp}$ & anticorrelated & Insufficient samples & Not obvious & No correlation with $\mathrm{Kp}$ or Ap & Insufficient sample \\
\hline
\end{tabular}

lightning generated causative spherics are marked by arrows. Whistler-mode waves were believed to be the most probable modes for the events reported here. Two probable source mechanisms, magnetosheath lion roar and lightening generated whistlers are discussed to explain the generation of these ELF whistlers.

Lion roar are wide band electromagnetic signals having a frequency below $120 \mathrm{~Hz}$ that occur in dayside magnetosheath. Magnetosheath lion roar have a field strength $\sim 0.1 \mathrm{nT}$ and the signal duration of $1 \mathrm{~s}$ (Smith et al., 1967; Smith and Tsurutani, 1976; Zhang et al., 1998; Baumjohann et al., 1999). Based on the frequency range and dayside occurrence of the observed ELF whistlers, Sentman and Ehring (1994) suggested that they were induced by lion roars that entred the Earth-ionosphere waveguide. However, ELF whistlers observed on the ground are coherent and narrowbanded, whereas lion roar emissions are incoherent and contain a band of frequencies, thus, creating a confusion how magnetosheath lion roars could be converted into the ELF whistlers (Wang et al., 2005). Also the lion roars are in the lower ELF range while our event in the study is much higher. Thus, magnetosheath lion roars have great deficits and are likely not the source of ELF whistlers observed at our low latitude ground station Jammu.

Sentman and Ehring (1994), Wang et al. (2005) and Kim et al. (2006) have reported ELF events with a long duration and a very high dispersion of about $5000 \mathrm{~s}^{1 / 2}$ and questioned how lightening generated whistler could generate such ELF 
events. However, the events observed at our station have a duration of only 0.5 to $3.5 \mathrm{~s}$ with dispersion lying between 68 to $252 \mathrm{~s}^{1 / 2}$. It is worthwhile to mention here that the dispersion of whistlers usually recorded in various low latitude ground stations in India are less than $25 \mathrm{~s}^{1 / 2}$ (Somayajulu et al., 1972) and high dispersion whistlers are mostly middle and high latitude whistlers (Helliwell, 1965). Hence, it may be inferred that the high dispersion whistlers in Fig. 1 are middle and high latitude whistlers which travelled to the observing station Jammu via Earth-ionosphere waveguide space-mode propagation. The various nose-extension methods have been developed for determining the nose frequency of non-nose whistlers (the whistlers which does not exhibit nose in its frequency-time dynamic spectra) since the discovery of whistlers (Bernard, 1973). In order to determine the propagation path of ELF whistlers shown in Fig. 1, we have applied the nose extension method of Dowden and Allcock (1971). From the computations we find that these whistlers have propagated along the geomagnetic field line in different ducts corresponding to L-values of 3.25 to 6.52 as shown in Table 1 which corresponds to high and mid latitude. For the reported ELF whistlers $L>3.25$ implies that some of the waves may have propagated through ducted mode propagation along higher L-values compared to low L-value ( $L=1.17$ ) of our observing station Jammu and after exiting from the duct, they penetrate the ionosphere and are trapped in the Earth-ionosphere waveguide. The wavenormal at the entrance into the waveguide is such that they propagated towards the equator and are received at the low latitude station Jammu (Singh et al., 2004b). Table 1 clearly shows that there exists different ducts $(L \sim 3.25-6.52)$ in the high latitude ionosphere and it is also evident that different whistler components of ELF whistlers have propagated along the geomagnetic field lines in closely spaced different ducts. Since our ELF whistlers are received during daytime on 4 March 1998, it may be suggested that the whistler components of ELF whistlers have propagated through different paths in the equatorial anomaly region of the ionosphere (Hayakawa and Tanaka, 1978; Singh and Hayakawa, 2001). Lightning generated whistlers observed on the Earth have a dispersion lying between $8-400 \mathrm{~s}^{1 / 2}$ and an event duration of a few seconds (Helliwell, 1965) and follow the Eckersley law. Our observed ELF whistlers have dispersion varying between $68 \mathrm{~s}^{1 / 2}$ to $252 \mathrm{~s}^{1 / 2}$ and event duration of 0.5 to $3 \mathrm{~s}$, and follow the Eckersley law as well. The corresponding lightning generated causative spherics are also shown by arrow in Fig. 1a, which supports the idea that the ELF events are lightning generated. Thus, our dispersion analysis shows that the ELF whistler observed at Jammu are lightning generated whistler during a geomagnetic disturbed day $\left(\sum \mathrm{Kp}=17_{+}\right)$.

According to Helliwell (1965) whistlers cutoff frequencies vary over a wide range of values. The low frequencies cutoff ranges from a minimum of about $300 \mathrm{~Hz}$ to as high as $10 \mathrm{kHz}$. The upper cutoff may be as low as $2 \mathrm{kHz}$ and as high as $40 \mathrm{kHz}$, as the upper cutoff of the equipment. In gen- eral, most whistlers lie between frequency range $2 \mathrm{kHz}$ and $10 \mathrm{kHz}$ whereas ELF whistlers observed in Jammu lie almost in ELF range between $\sim 400 \mathrm{~Hz}$ and $\sim 3.2 \mathrm{kHz}$. The whistler traces observed in our ELF whistlers pass through the waveguide cutoff frequency with almost no measurable decrease in intensity. Especially interesting is the occurrence of some whistlers showing a marked cutoff around waveguide cutoff frequency at $1.6 \mathrm{kHz}$ together with others in the same run that shows a much lower cutoff. Several factors may affect the observed cutoff frequencies of whistlers. Both the upper and lower cutoff is affected by the source spectrum and by the properties of the Earth-ionosphere waveguide. In addition, the lower cutoff is affected by ion resonances and upper cutoff by collisional absorption in the ionosphere, by duct properties and by thermal (Landau) damping near the top of the path (Helliwell, 1965). In the light of all the above factors involved in the cause of upper and lower frequencies of whistlers, it seems that the upper and lower cutoff frequencies in our observed ELF whistler at Jammu be limited as a result of the source mechanism only (Helliwell, 1965). It may be possible that the energy of a source lies only in the frequency range of the observed ELF whistlers without any attenuation.

Further, in addition to our ELF whistler event, we have also observed a trace of discrete riser (precursor) emission triggered almost from the upper frequency part of whistler traces (Fig. 1b: Group B). This could be explained due to nonlinear cyclotron resonance between whistler mode waves and high energetic particles. Normally such a type of ELF whistlers and precursors emissions observed during daytime are not transmitted to the ground due to heavy absorption in the lower ionosphere and large wave normal angles associated with them. However, some Japanese workers have observed large number of whistlers during daytime and interpreted their propagation to ground in terms of equatorial anomaly (Hayakawa and Tanaka, 1978). It is possible that the ELF/VLF waves recorded on 4 March 1998 at Jammu may have propagated under the influence of equatorial anomaly. This is the first of such an observation which has been recorded at a low latitude station and this phenomenon has not been discussed and explained theoretically earlier by any of the workers. For the complete understanding of such type of events more rigorous observation would be required and also a theoretical modelling is to be developed. So in the present state of knowledge, it is difficult to understand these phenomena completely and further study is needed.

\section{Conclusion}

Perhaps the most intriguing aspect of these low frequency whistlers confined to ELF-band is that they are widely distributed across the globe with a significant difference that the duration is very small. As for source of mechanism is 
considered magnetosheath lion roar have great deficit and is likely not the source of ELF whistler reported here. Under normal plasma conditions, observed dispersion of ELF whistler is shown only by lightning generated whistler events and have smaller event duration that is expected for low frequency of VLF whistlers. Thus, lightning seems to be the dominant source for the ELF whistlers reported here. This result may suggest that they are ducted over large regions but the lack of co-incidental observations discussed above does not support this notion. Similar ELF events in other stations relatively close to our low latitude site, Jammu and the investigation of long term variation would help to elucidate the potential role of multi-ion plasma in converting lightning generated whistlers into the observed ELF whistlers. The work reported here could invoke the interest of the scientific society again in ELF events and may contribute in resolving the mystery of the ELF whistlers.

Acknowledgements. The work is partially supported by the Department of Science and Technology, New Delhi, Government of India under SERC scheme and partly by ISRO, Bangalore. K. K. Singh thanks DST, New Delhi for award of FASTTRACK Fellowship. We are thankful to the reviewer for their valuable comments and suggestions to improve the quality of the paper.

Topical Editor R. Nakamura thanks one anonymous referee for her/his help in evaluating this paper.

\section{References}

Angerami, J. J. and Thomas, J. O.: Studies of planetary atmosphere: The distribution of ions and electrons in the Earth's exosphere, J. Geophys. Res., 69, 4537-4560, 1964.

Baumjohann, W., Treumann, R. A., Georgescu, E., Haerendel, G., Fornacon, K.-H., and Auster, U.: Waveform and packet structure of lion roars, Ann. Geophys., 17, 1528-1534, doi:10.1007/s00585-999-1528-9, 1999.

Bernard, L. C.: A new nose extension method for whistlers, J. Atoms. Terr. Phys., 35, 871-880, 1973.

Carpenter, D. L. and Anderson, R. R.: An ISEE/Whistler Model of Equatorial Electron Density in the Magnetosphere, J. Geophys. Res., 97, 1097-1108, 1992.

Cummer, S. A., Inan, U. S., Bell, T. F., and Barrington-Leigh, C. P.: ELF radiation produced by electric currents in sprite, Geophys. Res. Lett., 25, 1281-1285, 1998.

Dowden, R. L. and Allcock, G. M.: Determination of nose frequency of non-nose whistlers, J. Atoms. Terri. Phys., 33, 11251129, 1971.

Hayakawa, M. and Tanaka, Y.: On the propagation of low latitude whistlers, Rev. Geophys. Space Phys., 16, 111-123, 1978.

Heacock, R. R.: Whistler-like pulsation events in the frequency range 20-200 Hz, Geophys. Res. Lett., 1, 77-79, 1974.
Helliwell, R. A.: Whistlers and Related Ionospheric Phenomena, Stanford Univ. Press, Stanford, California USA, 1965.

Hoffman, W. C.: The current-jet hypothesis of whistler generation, J. Geophys. Res., 65(7), 2047-2054, 1960.

Kim, H., Lessard, M. R., LaBelle, J., and Johnson, J. R.: Narrowband extremely low frequency (ELF) wave phenomena observed at South Pole Station, Geophys. Res. Lett., 33, L06109, doi:10.1029/2005GL023638, 2006.

Magunia, A.: The thunderstorm-driven diurnal variation of the ELF electromagnetic activity level, J. Atmos. Sol Terr. Phys., 56, 1683-1696, 1996.

O'Brien, B. J.: High-latitude geophysical studies with satellite Injun 3 3. Precipitation of electrons into the atmosphere, J. Geophys. Res., 69(1), 13-43, 1964.

Sazhin, S. S., Hayakawa, M., and Bullough, K.: Whistler diagnostics of magnetospheric parameter, a review, Ann. Geophys., 10, 293-308, 1992.

Sentman, D. D. and Ehring, D. A.: Midlatitude detection of ELF whistlers, J. Geophys. Res., 99, 2183-2190, 1994.

Singh, D. Singh, A. K., Patel, R. P., Singh, R., Singh, R. P., Veenadhari, B., and Mukherjee, M.: Thunderstorms, lightning, sprites and magnetospheric whistler-mode radio waves, Surv. Geophys., 29, 499-551, doi:10.1007/s10712-008-9053-z, 2008.

Singh Ashutosh K., Patel, R. P., Singh, R., Singh, K. K., and Singh, A. K.: Characteristics of discrete VLF falling-tone chorus emissions observed at low latitude ground station Jammu, Earth Planet Space, 61, 1179-1183, 2009.

Singh, R. P., Singh, A. K., and Singh, D. K.: Plasmasphere parameters from whistler spectrograms: a review, J. Atmos. solar-Terr. Phys., 60, 495-508, 1998.

Singh, B. and Hayakawa, M.: Propagation modes of low- and verylow-latitude whistlers, J. Solar-Terr. Phys., 63, 1133-1147, 2001.

Singh, K. K., Singh, R., Singh, R. P., and Shyampati: Hisslers: Quasi-periodic VLF noise forms observed at low latitude ground station Jammu ( $L=1.17)$, Geophys. Res. Lett., 31, L19802, doi:10.1029/2004 GL-020468, 2004a.

Singh, R. P., Singh, R., Lalmani, Hamer, D., and Lichterberger, J.: Application of matched filtering to short whistlers recorded at low latitudes, J. Atmos. solar-Terr. Phys., 66, 407-413, 2004b.

Smith, E. J., Holtzer, R. E., Macleod, M. G., and Russell, C. T.: Magnetic noise in the magnetosheath in the frequency range 3$300 \mathrm{~Hz}$, J. Geophys. Res., 72, 4803-4813, 1967.

Smith, E. J. and Tsurutani, B. T.: Magnetosheath lion roars, J. Geophys. Res., 81, 2261-2266, 1976.

Somayajulu, V. V., Rao, M., and Tantry, B. A. P.: Whistlers at low Latitudes, Indian J. Radio Space Phys., 1, 102-118, 1972.

Wang, Y.-C., Wang, K., Su, H.-T., and Hsu, R.-R.: Low-latitude ELF whistlers observed in Taiwan, Geophys. Res. Lett., 32, L08102, doi:10.1029/2005GL022412, 2005.

Zhang, Y., Matsumoto, H., and Kojima, H.: Lion-roars in the magnetosheath: The Geotail observations, J. Geophys. Res., 103, 4615-4626, 1998. 